\title{
Condiciones dignas de la labor docente del siglo XXI, un derecho inalienable, no una opción
}

\section{Dignified Teacher Working Conditions in the 21st Century, an Inalienable Right, not an Option}

\author{
Guiselle María Garbanzo Vargas ${ }^{l}$ \\ Escuela de Administración Educativa \\ Universidad de Costa Rica \\ San José, Costa Rica \\ gmgarban@gmail.com
}

Recibido 14 de abril de 2011 - Aceptado 26 de agosto de 2011

\begin{abstract}
Resumen. Se desarrolla el tema de las condiciones dignas de la labor docente en el desafiante siglo XXI. El objetivo recae en realizar una contextualización de la sociedad emergente en el siglo XXI, en general; así como plantear una revisión, en América Latina, del contexto educativo y de las mismas condiciones laborales del profesional de la educación. Otro objetivo es revisar las condiciones laborales en que se encuentran los educadores en distintos sistemas educativos, incluyendo realidades de Europa y América Latina, entre otras. En las principales ideas que se abordan, se define el perfil del educador de siglo XXI en comparación con el educador de la sociedad tradicional y se desarrollan algunos indicadores de consenso en distintas realidades educativas, así como su integración en tres grandes dimensiones: cognitivas, operativas e institucionales. Sobresale, entre las principales conclusiones, la determinación de políticas públicas que respalden las condiciones laborales en las que se desempeñe el docente de manera digna, lo cual se considera fundamental para una educación inclusiva de calidad, en armonía con las exigencias de la desafiante sociedad emergente. Concluye la autora que este tema es inacabado y debe ser una constante en las agendas educativas de las distintas sociedades.
\end{abstract}

Palabras claves. Dignidad docente, condiciones laborales del docente, educación, profesional de la educación.

\footnotetext{
Licenciada en Ciencias de la Educación con énfasis en Orientación de la Universidad de Costa Rica (UCR) y en Administración con énfasis en Recursos Humanos de la Universidad Nacional (UNA). Tiene una Maestría en Administración Educativa de la Universidad de Costa Rica (UCR) y es Doctora en el Programa de Doctorado Latinoamericano en Educación de la Universidad de Costa Rica. Actualmente, se desempeña como directora, docente e investigadora en la Escuela de Administración Educativa de la Facultad de Educación de la Universidad de Costa Rica (UCR) y es coordinadora del Posgrado en Ciencias de la Educación con énfasis en Administración Educativa de la Universidad de Costa Rica (UCR) y Directora de la Revista Gestión de la Educación. Ha publicado varios artículos en el campo de la educación superior pública y de la administración de la educación
} 


\begin{abstract}
The author develops the topic of appropriate teacher working conditions in the challenging $21^{\text {st }}$ Century. The objective is the contextualization of the emergent society in the $21^{\text {st }}$ Century; propose a review of the Latin American educational context and the working conditions of education professionals. Another objective is to review the working conditions of teachers in different educations systems, including Europe and Latin America, among others. The profile of the 21st Century educator is defined in this paper, in comparison to teachers in the traditional society. In addition, this paper includes some indicators of consensus in different educational realities, and their integration in the cognitive, operational and institutional areas. The main conclusions include the necessity of policy-making to support dignified teacher working conditions, which is basic for an inclusive education of quality, in accordance with the demands of a challenging emerging society. The author concludes that this issue does not come to an end; it must always be in the educational agenda of society.
\end{abstract}

Keywords. Teacher's dignity, teacher-working conditions, education, education professionals.

\title{
Contextualización:
}

En el siglo XXI, a la humanidad le corresponde vivir en una sociedad altamente compleja, marcada por la información y la comunicación, cuyas características han estremecido y transformado el ritmo y las formas de operar de la sociedad como un todo, donde las barreras no existen entre naciones y las redes de conexión son una realidad indispensable para el desarrollo competitivo, social y personal al que aspira.

La base material de la sociedad se ha visto modificada, aceleradamente, por diferentes razones. Una de ellas es el marcado énfasis en las tecnologías de la información, el cual ha permeado todas las estructuras sociales. Los nuevos parámetros de competitividad han requerido de las organizaciones un rol más descentralizado, flexible e interconectado. El individualismo, a criterio de Castells (2007), también se incrementa y las sociedades han pasado a funcionar de forma interdependiente. El tiempo real no es un límite, la virtualidad emerge como un orden organizacional y social.

Los nuevos desafíos de la sociedad generan nuevos problemas, formas de interacción y la búsqueda de nuevos enfoques epistemológicos. Constituyen una revolución en las formas de pensamiento, la cual deja atrás tradicionales construcciones cognitivas, para asumir un pensamiento transdisciplinario, en el marco complejo de la sociedad emergente. Traen implícito un repensar de la concepción del hombre articulado con un nuevo paradigma de construir y producir el conocimiento. Uno de los principales cambios que se experimentan es la modificación de los espacios donde se produce el conocimiento científico en el sistema del saber humano, lo que, a criterio de Aguerrondo (2007), conduce a la construcción de un saber nuevo, articulado con las soluciones teóricas innovadoras, como el enfoque de la complejidad.

Las organizaciones, en el nuevo orden globalizado, se han enfrentado a diferentes transformaciones; actúan en escenarios variados, complejos y amplios; enfrentan una especie de metamorfosis organizacional, que las dota de oportunidades, amenazas, riesgos y contingencias, lo que propicia el renacimiento de nuevas tendencias y enfoques de la administración moderna (Chiavenato, 2002). La sociedad emergente se encuentra sumergida en la complejidad de sus interacciones y sus formas de competir. Este contexto también trae desafíos para la persona, indistintamente de su ubicación social: se requiere de individuos con una visión crítica e interdisciplinaria, lo que ha hecho repensar sus mismos sistemas educativos en busca de la competitividad que la sociedad espera de ellos, de manera que la cobertura educativa, la calidad y la inclusión de una educación para todos, sea una realidad. 


\section{La educación en el nuevo contexto social}

América Latina, a pesar de las limitaciones de la región y siendo la más desigual del Orbe, presentó, en la década de los ochenta, un esfuerzo en cobertura educativa (VII Reunión del Comité Regional Intergubernamental del Proyecto Principal de Educación. Declaración de Cochabamba, 2001). A su vez, en la década de los noventa, en busca de un nuevo desarrollo educativo, a la luz de las demandas del momento, experimentó un período de sostenibilidad y debió ofrecer respuestas a fenómenos como multiculturalidad, equidad social, reformas, políticas educativas y democratización política; todos ellos propios de un mundo exigente, según los desafíos sociales emergentes del nuevo orden social.

El siglo XXI llegó sin que aún se hubieran concluido y resuelto importantes problemas educativos, como mejorar o lograr mejor calidad en los sistemas, el desarrollo de la educación de manera integral, la situación laboral del docente, el acceso educativo y una educación de calidad para todos (VII Reunión del Comité Regional Intergubernamental del Proyecto Principal de Educación. Declaración de Cochabamba, 2001).

A pesar de los esfuerzos realizados, el inicio del nuevo siglo evidencia una serie de tareas educativas -relacionadas con la eficiencia y calidad de los sistemas de enseñanza aprendizaje- sin concluir. Este tema fue ampliamente analizado por los Ministros de Educación de América Latina y el Caribe, en la VII Reunión del Comité Regional Intergubernamental del Proyecto Principal de Educación, llevada a cabo en México, en el 2001. Se reconoció que el nuevo siglo inició con importantes tareas pendientes en la región, tales como el acceso a una educación de calidad para todos, así como el mejoramiento de las condiciones laborales del profesional en educación.

El siglo XXI arriba con determinación clara de mejorar, en forma paralela con el mejoramiento de la labor docente, la calidad educativa y la eficiencia de los sistemas educativos; reconoce que la labor docente constituye un recurso estratégico en cualquier sistema educativo para lograr los fines y objetivos de la educación propuestos, el logro de una educación inclusiva y de igual calidad para todos, según sus particularidades, siguen siendo retos claramente determinados. En el actuar de la sociedad emergente, el acceso a la educación de calidad para todos es fundamental e impostergable para los ciudadanos. Este noble proceso educativo, en su operacionalización, está en manos de los profesionales en educación, quienes requieren para un óptimo desarrollo condiciones laborales adecuadas, con el fin de que el proceso de enseñanza se ofrezca de forma eficiente y eficaz; aspecto que aún no se considera como un logro acabado en distintitos sistemas educativos, principalmente, en América Latina.

Es así como el nuevo siglo llega con un vertiginoso desarrollo de las ciencias y las nuevas tecnologías, escenarios con complejos y enormes desafíos y oportunidades para la región. El contexto se hace cada vez más plural y diverso, lo cual implica docentes con competencias para trabajar en un mundo multicultural, altamente complejo y transdisciplinario, que se desarrolla de forma constante. Estas condiciones diversas no son ni pueden verse en los albores del siglo XXI como amenazas; por el contrario, son recursos de aprendizaje estratégicos.

Se experimentan, en este contexto, dificultades para concebir y tejer una visión holística de las reformas educativas y los cambios necesarios para que la educación opere integralmente, no de forma compartimentada en sus distintos espacios y ejes estratégicos. Es indispensable una visión de conjunto que abarque el mediano y largo plazo.

Esta coyuntura lleva a los distintos actores sociales involucrados en el desarrollo humano y social a repensar la educación y queda claro que sin educación no hay desarrollo humano viable. La 
educación por sí misma no resuelve los problemas que afligen la sociedad, ni hace virar el rumbo social hacia el crecimiento; sin embargo, la educación es una disciplina estratégica para el desarrollo humano, razón por la cual es parte prioritaria -al menos en las agendas gubernamentales- en las políticas públicas. Es importante reconocer que hay muchos actores sociales que intervienen para hacer del proceso educativo un espacio con mejores rangos de calidad y equidad.

La educación es un derecho fundamental de toda persona, por lo tanto, es obligación de los estados asegurar una educación adecuada para toda la población. Es un medio indispensable en la búsqueda de la paz, la justicia social y la libertad. Lleva implícito un rol esencial en el desarrollo continuo de la persona y de la sociedad como un todo, pues permite aspirar a un crecimiento humano cada día más armonioso, justo e inclusivo (Delors et al., 1996).

La educación trae consigo el reto ético, fortalecedor de los distintos valores y actitudes en los que se respalda el actuar de la persona. Aporta, también, un sentido altruista, inspirador, incorruptible y humanista en el componente económico y en el plano social. En el individual, favorece el asumir el desafío referente al pleno desarrollo de la personalidad de quienes asumen el rol de educandos, de forma que se atienda, adecuadamente, el desarrollo de sus propias aptitudes, de acuerdo con su vocación y lo concerniente al sentido mismo de la vida. Incluye, además, el respeto a sí mismo, al tomar en cuenta el cultivo de la voluntad de bien común y la preparación para una vida en familia de manera digna. Asume relevancia, también, el reto ético a lo interno. Al respecto, es un deber del Ministerio proveer a los educadores los medios necesarios para llevar con éxito sus obligaciones en la consecución de la política educativa (Ministerio de Educación Pública, 1994).

En la medida en que la educación es un derecho humano, es responsabilidad del Estado otorgar condiciones aceptables y dignas al profesional en educación. No puede concebirse la implementación de las reformas educativas sin contemplar, en ellas, la necesidad de velar por las condiciones laborales del docente.

Las reformas educativas y las condiciones dignas laborales del educador representan un binomino conceptual y práctico indisoluble. En sociedades en las que las brechas de desigualdad, pobreza y acceso - como lo es América Latina- evidencian, de forma indirecta, una importante violación al derecho humano de acceder a la educación, y en las condiciones laborales del educador, el Estado debe asumir, responsablemente, la promoción de políticas educativas necesarias de forma articulada con las condiciones profesionales dignas del docente. Al respecto, hay que tener en cuenta que estas políticas por sí mismas no llevan a nada, no son un fin, son un medio que viene a representar una importante base social para orientar y respaldar el quehacer educativo en sus distintas áreas.

Dentro de este quehacer educativo asume relevancia la educación como un proceso mediante el cual se potencia el desarrollo humano, el progreso espiritual, social, cultural y económico de las sociedades, lo cual lleva implícita la capacidad de inculcar el respeto por los derechos humanos en forma profunda. En este sentido, la UNESCO-OIT (1996) considera que la educación debería marcar, como objetivo, desde temprana edad, a la persona en la escuela, de la siguiente manera:

(...) el pleno desarrollo de la personalidad humana y el progreso espiritual, moral, social, cultural y económico de la comunidad, así como inculcar un profundo respeto por los derechos humanos y las libertades fundamentales (...). (p. 24)

En este marco conceptual, la educación aspira a la formación de personas conscientes de sus deberes, derechos, libertades, aunada al respeto de la dignidad humana y con sentido de amor a su 
patria. Es potencializar el desarrollo de las habilidades cognitivas, morales, éticas, espirituales de los seres humanos en busca del desarrollo humano y social; perfil del ciudadano por el cual debe luchar la sociedad.

La educación, para operacionalizar sus objetivos y fines, se encuentra ante una serie de retos, a los cuales debe hacer frente (VII Reunión del Comité Regional Intergubernamental del Proyecto Principal de Educación. Declaración de Cochabamba, 2001):

- Toda Política Educativa, con aspiraciones a trascender más allá de períodos gubernamentales, requiere apoyarse y fundamentarse en el marco jurídico que en su nación particular rige la educación. Debe contemplar lo económico y social.

- Las nuevas tendencias mundiales en la actual sociedad de la información enrumban la educación en cuatro ejes de desarrollo sostenible, que los sistemas educativos deben considerar en sus políticas educativas: la sostenibilidad ambiental, social y política, del recurso humano y finalmente la sostenibilidad económica y productiva.

- Estos ejes de sostenibilidad interactúan de forma paralela y por sí mismos. Entre ellos se enfrentan al desafío ambiental, económico, social y la sostenibilidad del personal docente. También, se considera un desafío lo ético, que asume un papel transversal, subyacente como denominador común a todos.

Son muchos los componentes que hacen de un proyecto educativo el más valioso para las agendas gubernamentales. En distintos debates se ha reconocido esta importancia, así lo hicieron ver los Ministros de Educación de América Latina y el Caribe, reunidos en Cochabamba, México, en convocación de la UNESCO (VII Reunión del Comité Regional Intergubernamental del Proyecto Principal de Educación. Declaración de Cochabamba, 2001).

Se pueden resumir algunos desafíos de la educación en América Latina; todos ellos esperan respuesta de los actores sociales involucrados en este proceso:

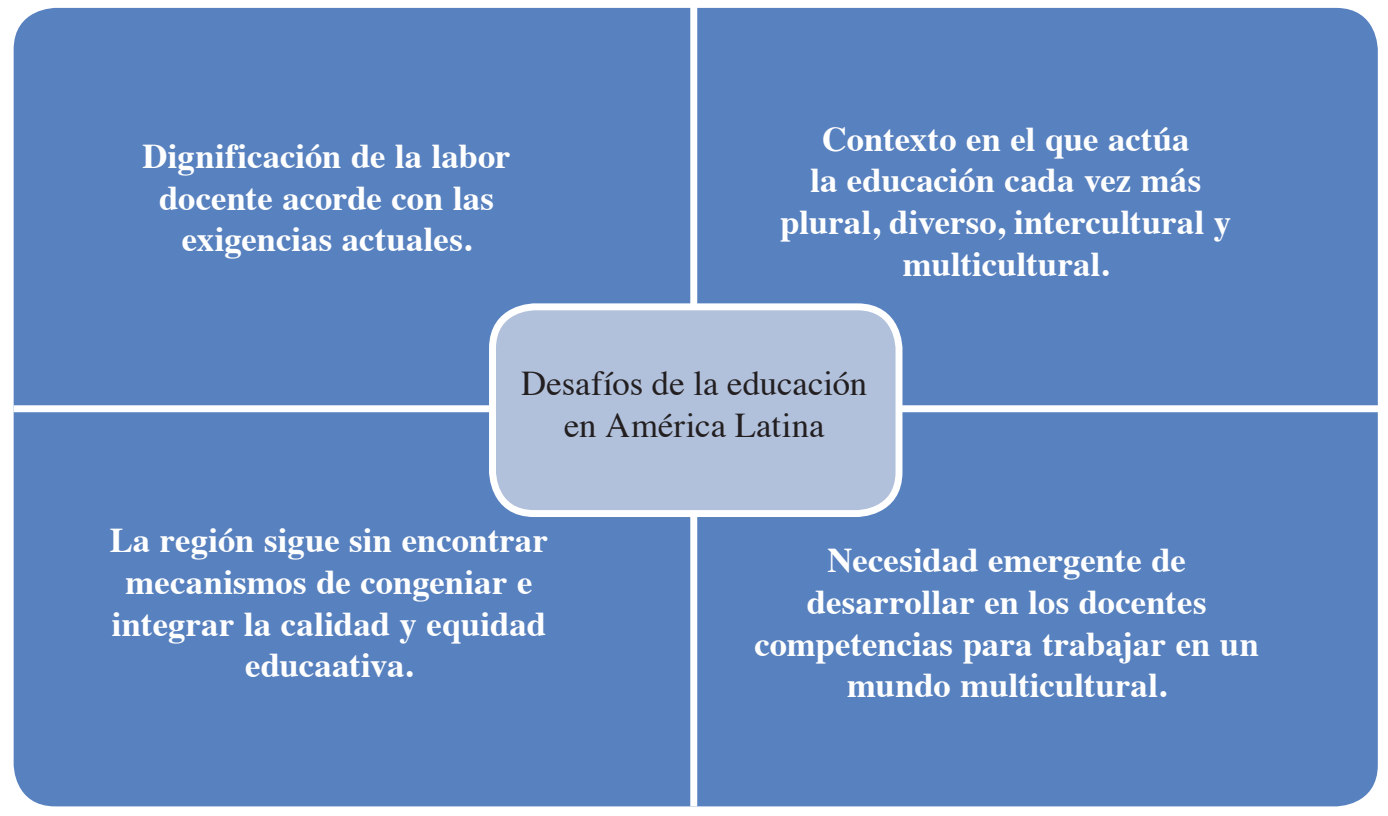




\section{El profesional en educación en los albores del siglo XXI}

En general, en los distintos debates en torno a la educación, hay claro consenso a nivel de las propuestas gubernamentales, en cuanto a que la educación y los profesionales en educación son estratégicos en el desarrollo humano y social. En el sistema educativo costarricense, esta concepción queda visualizada en su Política Educativa del siglo XXI, en la cual se concibe al educador como la figura clave en la transformación de la educación costarricense. Además, se reconoce que su profesionalismo y calidad de persona será determinante para operacionalizar el proyecto educativo en forma seria y responsable.

El plano político, en el que se mueven los profesionales en educación, asume un alto valor para que estos profesionales puedan liderar el proceso de enseñanza en forma cabal. Son muchos los elementos por considerar para aspirar, mediante los educadores, a las transformaciones que la sociedad requiere para mantenerse en planos globales competitivos. Dentro de estos elementos que toda política educativa debe considerar, se encuentran:

- Para estar al día con las exigencias que la nueva sociedad trae consigo, todo proceso de transformación, que los gobiernos lideran mediante sus sistemas educativos, debe hacerse de la mano con los actores del mismo proceso educativo. En este, sus docentes, mediante sus prácticas, determinan, en gran medida, los cambios.

- Para la ejecución de las políticas educativas, además de contar con un cuerpo docente idóneo que lidere los procesos de cambio, se requiere promover las condiciones adecuadas para que las instituciones educativas tengan espacios de aprendizaje dignos, aptos para la innovación y para los indispensables cambios pedagógicos, según las necesidades de los tiempos.

- Una educación de calidad se logra mediante las potencialidades y competencias del docente, el cual, no obstante el avance tecnológico, seguirá siendo insustituible.

- Toda transformación que suponen e implican las reformas educativas está de una u otra forma determinada por la preparación profesional y condiciones laborales del docente, donde cuentan aspectos como el desarrollo profesional, la remuneración y el aprendizaje permanente.

De esta forma, son las condiciones laborales del docente, así como las condiciones y la calidad en las que se da su formación académica y profesional, determinantes, en gran medida -no las únicas- del logro de una mejor calidad de la educación; por tanto, las variables asociadas a su quehacer merecen toda consideración, desde la política, social y educativa -según las transformaciones sociales requeridas-, hasta las que implican reformas educativas -para adaptarse a las necesidades de los tiempos-. Al respecto, Bruner (1988) se manifiesta en contra de la mediocridad y a favor de la excelencia, mejor rendimiento, mayor alfabetización, destrezas numéricas, verbales y mayor disciplina. Esta visión es digna de considerar, en cuanto a lo que produce, socialmente, la fuerza laboral docente; una razón más para prestar atención a la forma laboral en que opera.

En el nuevo contexto globalizado, indistintamente del sistema educativo, el rol docente ha pasado por un proceso en el que se ha repensado, según las exigencias del nuevo milenio. El docente de los actuales tiempos, en su ejercicio, debería actuar alejado de un rol tradicional 
como instrumentalizador del currículo, para pasar a protagonista de este, todo ello, en escenarios educativos donde el aula sea el centro de los cambios reales (Robalino, 2005). También, el rol de docente trasmisor de conocimientos debe quedar atrás para asumir uno de docente gestor del conocimiento.

Desde esta perspectiva, los desafíos de la sociedad emergente requieren un replanteamiento del rol del educador. Como actor social del proceso de enseñanza, deberá enfrentar y seguir actuando sobre transformaciones de las prácticas pedagógicas tradicionales, las cuales, tal y como se concibieron, ya no tienen cabida en la nueva sociedad de la información. Los educandos han cambiado también sus formas de adquirir el conocimiento; el nuevo orden mundial espera y le exige, al profesional en educación, un replanteamiento de sus formas de enseñar, pues debe liderar un nuevo proceso educativo a la luz de los nuevos tiempos.

En este proceso de replanteamiento de la práctica docente, las autoridades educativas tienen en sus manos el deber de suplirle una serie de necesidades para lograr armonizar su desempeño con las exigencias sociales: líneas de capacitación y educación continua de los docentes; iniciativas sostenibles en la medida en que tengan un carácter permanente y articulado con los responsables de los ministerios de educación y las instituciones formadoras de estos profesionales.

Todo cambio en las prácticas educativas aspira a una mejor calidad de la educación que se ofrece, y esta, en gran medida, está determinada también por la calidad del profesional en educación. La calidad de los educadores es un reto que los sistemas educativos en conjunto con las universidades formadoras de estos profesionales deben contemplar con visión de futuro. Conforme la sociedad cambia, los docentes deben ajustarse a estos vertiginosos movimientos, y esta brecha, cuando se genera, es una de las principales causas de los fracasos. En muchas ocasiones, estos grandes temas carecen de un anclaje entre sus componentes y son notorias las fisuras existentes.

El mejoramiento de la calidad del cuerpo docente es considerado por distintos sistemas educativos como prioritario en sus agendas políticas. Al menos en el Ministerio de Educación Pública de Costa Rica, la calidad del docente está dentro de las 10 líneas estratégicas de su plan de trabajo: es considerada clave para mejorar la calidad misma de la educación y vital para aspirar a la profesionalización y dignificación de los docentes (MEP, 2010).

El perfeccionamiento del recurso humano en educación busca elevar, consecuentemente, el nivel profesional, de manera que el desempeño, el compromiso y la motivación alcancen estándares altos, para lograr la transformación requerida por las exigencias sociales en función de la excelencia y la equidad hacia la búsqueda de una educación inclusiva para todos.

Los programas de actualización ofrecidos a los profesionales de la educación en servicio tienen como principios orientadores: brindar oportunidades de formación inicial, proporcionar oportunidades de capacitación a quienes no posean un nivel de formación aceptable para su óptimo desempeño y actualizar en prácticas pedagógicas de la postmodernidad.

\section{¿Qué se espera de la práctica del profesional en educación?}

Son variadas y complejas las demandas sociales a las que los profesionales en educación se enfrentan; y, muy exigentes las prácticas educativas que se esperan del educador, acordes con las 
necesidades de los nuevos tiempos. Todas las respuestas no vienen por sí mismas, requieren y se apoyan para su implementación de transformaciones fundamentales en las políticas educativas, que les permitan a los profesionales en educación enfrentar los desafíos en forma eficiente y eficaz. Estas poseen implicaciones en la disposición y condiciones en que los educadores operan en el sistema educativo en el cual actúan. El éxito de las políticas educativas depende, en gran medida, de las condiciones en que se desempeñan los educadores.

Se espera en los caminos del siglo XXI, un profesional en educación cada vez más flexible, conocedor de las diferentes disciplinas y con una preparación adecuada para dar respuesta desde diferentes fuentes del saber (MEP, 1994). El docente que el nuevo siglo reclama, debe ser poseedor de una visión de mundo holística, no compartimental, con una comprensión multidisciplinar e interdisciplinar y consciente de la complejidad social en la que la educación actúa. Son muchas las variables que desafían el contexto educativo emergente, entre las que se encuentran lo económico, lo político, lo tecnológico, lo cultural y lo social.

El docente, según las nuevas demandas, requiere desarrollar competencias para trabajar en redes de comunidades prácticas, donde la barrera del centro educativo sea ampliamente superada como por una acción en una sociedad interconectada. Los educadores, además de trabajar de forma interconectada, deben apropiarse de las tecnologías de la información y la comunicación como herramienta útil y no pasiva para la enseñanza. Es un docente con un amplio sentido ético en su actuar y dispuesto a incursionar en los cambios requeridos de forma inmediata. Los profesionales en educación, al igual que los de las distintas disciplinas, deben adquirir una actitud incasable hacia el crecimiento profesional, de manera que sus conocimientos sean constantemente actualizados. Aunado a ello, la pasión por el saber se considera una condición indispensable.

En el mundo intercultural, es de rigor un adecuado perfil profesional para trabajar en la diversidad en una sana convivencia, donde el respeto prevalezca. Le corresponderá laborar en ambientes cambiantes, para lo que deberá tener la capacidad de repensar sus formas de enseñanza a la luz de las nuevas formas de aprender del estudiantado. En este contexto desafiante, la formación inicial y la continua conforman un binomio indisoluble, el cual no tiene opción de minimizar. De igual manera, debe considerar la incorporación de la práctica profesional en procesos de investigación.

El profesional en educación no debe ser un espectador de la generación del conocimiento, su papel como formador en los procesos educativos le requiere asumir una función protagónica en los distintos procesos de investigación, mediante los cuales genere el conocimiento. Sobre este tema, los Ministros de Educación de América Latina y el Caribe, en la Declaración de Cochabamba (VII Reunión del Comité Regional Intergubernamental del Proyecto Principal de Educación. Declaración de Cochabamba, 2001), consideran que:

(...) La formación docente demanda ser reconceptualizada con un enfoque sistémico, que integre la formación inicial con la formación continua, la participación efectiva en proyectos de mejoramiento, la generación en los centros educativos de equipos de trabajo docente y la investigación en una interacción permanente (...). (p. 115) 
Los retos intelectuales y éticos que recibieron al siglo XXI se encontraron con modelos pedagógicos que ya no son viables: a los docentes ya no les pertenece el conocimiento, este circula en todas partes; sin embargo: "(...) es aún del maestro la capacidad para enseñar a aprender, a mirar, a leer e interpretar la realidad, el propio yo (...)" (Bazarra, Casanova y García, 2005, p. 37).

En respuesta a esta coyuntura, para los educadores, son retos del siglo XXI: trabajar mediante el establecimiento de redes de apoyo pedagógico, establecer comunidades académicas interconectadas, que rompen las barreras físicas y de tiempo. El docente de este siglo, sabe que así como la postmodernidad trajo consigo nuevas formas de aprendizaje, estas son paralelas al repensar de los escenarios en que se mueve, desempeña y aprende. La nueva sociedad hace emerger espacios colectivos mediante redes para compartir y aprender los saberes requeridos para su eficiente desempeño, en un mundo en constantes transformaciones sociales.

Para poder llevar a cabo los cambios fundamentales en las formas de enseñar requeridas, debido a las nuevas formas de aprender de los estudiantes, es necesario contar con espacios pedagógicos que faciliten estos procesos. Los espacios y formas pedagógicas tradicionales no responden del todo a las exigencias educativas de hoy. Los paradigmas educativos emergentes de base epistemológica constructivista como el psicogenético y el sociocultural son una opción para este cambio. Como consecuencia, se deben repensar la infraestructura educativa, el tamaño de los grupos y las tecnologías educativas (Flores, 2000).

Hay que democratizar el uso de las tecnologías. El mundo apunta a moverse a través de la tecnología, mediante redes. Serán los docentes parte de comunidades educativas, para lo que necesitan que el uso de la Internet y de las tecnologías sean de fácil acceso. El educador del siglo XXI se moverá, rápidamente, a estos paradigmas, y este avance vertiginoso de la labor docente se concibe solo si sus condiciones laborarles son adecuadas (VII Reunión del Comité Regional Intergubernamental del Proyecto Principal de Educación. Declaración de Cochabamba, 2001).

Los nuevos lineamientos en materia curricular, para su eficiente desempeño, requieren de una inversión responsable de oportunidades de desarrollo profesional y competencias. El profesional en educación necesita de mejores y adecuadas oportunidades para integrar en el aula la teoría y la práctica. Estas oportunidades de desarrollo profesional dejan atrás un enfoque tradicional exclusivo dentro del centro educativo u otras instancias físicas, para pasar a complementarse con enfoques bimodales (Darling-Hammond y McLaughlin, 2003). A manera de ejemplo, a mediados del siglo pasado, para ser educador, se solicitaba a las personas que presentaran una certificación de que sabían leer y escribir, además, que estuvieran vestidas con aseo y calzados; sin embargo, este perfil hoy no es ni pensado como una opción, la práctica docente ha repensado su rol de forma drástica, como consecuencia de las demandas de la sociedad emergente, tal y como se detalla en la siguiente tabla: 


\section{URL: http://www.una.ac.cr/educare}

Tabla 1

El educador según exigencias sociales

\begin{tabular}{|c|c|}
\hline Sociedad tradicional & Sociedad emergente \\
\hline Conocimiento lineal & Poseedor de conocimiento interdisciplinario \\
\hline $\begin{array}{l}\text { Conocedor de una misma disciplina, campo de acción } \\
\text { desde el cual actúa }\end{array}$ & $\begin{array}{l}\text { Cada vez más flexible, conocedor de las diferentes disciplinas } \\
\text { y con una preparación adecuada, para dar respuesta desde } \\
\text { diferentes fuente del saber }\end{array}$ \\
\hline $\begin{array}{l}\text { Desconocimiento de la diversidad y la interculturalidad en } \\
\text { forma prioritaria }\end{array}$ & $\begin{array}{l}\text { Capacitado para trabajar desde y para la diversidad, tolerancia } \\
\text { y la interculturalidad }\end{array}$ \\
\hline $\begin{array}{l}\text { Conocimiento en el cual se formaba, como facilitador de su } \\
\text { labor por amplios períodos }\end{array}$ & $\begin{array}{l}\text { Poseedor de cualidades intelectuales, morales y competencias } \\
\text { profesionales adecuadas a las demandas de la postmodernidad }\end{array}$ \\
\hline Sin debate permanente sobre la formación continua & Constante desarrollo profesional \\
\hline Alejado de los procesos de investigación & $\begin{array}{l}\text { Competencias de investigador y capacitado para poner en } \\
\text { práctica el nuevo conocimiento derivado de la investigación, } \\
\text { protagonista de la generación del conocimiento y no un } \\
\text { espectador }\end{array}$ \\
\hline $\begin{array}{l}\text { Su labor considerada un apostolado de sacrificio fuera de su } \\
\text { jornada laboral }\end{array}$ & $\begin{array}{l}\text { Profesional al que se le respeta su jornada laboral, y se le } \\
\text { exonera de actividades fuera de sus funciones profesionales }\end{array}$ \\
\hline $\begin{array}{l}\text { Práctica pedagógica exclusiva limitada a los recursos } \\
\text { pedagógicos tradicionales }\end{array}$ & $\begin{array}{l}\text { Mayor presencia en la práctica pedagógica de las tecnologías } \\
\text { de la información y la comunicación en los procesos de } \\
\text { enseñanza y aprendizaje }\end{array}$ \\
\hline Visión de mundo limitada a un contexto específico & $\begin{array}{l}\text { Abierto al progreso, con amplia visión de mundo y proyec- } \\
\text { ción futura }\end{array}$ \\
\hline Nuevas tecnologías sin un lugar en los espacios educativos & $\begin{array}{l}\text { Con elevados conocimientos de la educación virtual y manejo } \\
\text { de variadas plataformas tecnológicas }\end{array}$ \\
\hline $\begin{array}{l}\text { Sin apropiación de las nuevas tecnologías, las cuales no se } \\
\text { perciben prioritarias ni como una necesidad }\end{array}$ & $\begin{array}{l}\text { Apropiación de las nuevas tecnologías que la postmodernidad } \\
\text { pone al servicio de la enseñanza }\end{array}$ \\
\hline Sin concepción cultural de publicar su pensamiento & Con competencias para escribir y publicar su pensamiento \\
\hline Con manejo del mundo en forma segmentada & $\begin{array}{l}\text { Interconectados con comunidades de aprendizaje } \\
\text { corporativas, mediante redes de aprendizaje }\end{array}$ \\
\hline $\begin{array}{l}\text { Concepción de la investigación como innecesaria para el } \\
\text { desempeño docente }\end{array}$ & $\begin{array}{l}\text { Competitivo, un profesional capaz de gestar y liderar } \\
\text { procesos de investigación, teniendo como punto de partida su } \\
\text { desempeño pedagógico }\end{array}$ \\
\hline Capacidad crítica, reflexiva y analítica & $\begin{array}{l}\text { Con elevadas competencias para la reflexión, la crítica y el } \\
\text { análisis }\end{array}$ \\
\hline Visión cultural, disciplinaria & $\begin{array}{l}\text { Amplia visión teórico-práctica intercultural, interdisciplinaria } \\
\text { y multidisciplinaria }\end{array}$ \\
\hline $\begin{array}{l}\text { Tecnología limitada a la tenencia de apartados para una } \\
\text { actividad determinada }\end{array}$ & $\begin{array}{l}\text { La tecnologías de la información como propiciadora, en la } \\
\text { práctica docente, de una ruptura en el tiempo y el espacio, } \\
\text { dentro de los procesos de aprendizaje que desarrolla }\end{array}$ \\
\hline $\begin{array}{l}\text { Enfoques pedagógicos tradicionales conductistas y } \\
\text { trasmisores de información }\end{array}$ & $\begin{array}{l}\text { Enfoques pedagógicos basados en la interacción entre ambas } \\
\text { partes, constructivista, humanista y racionalista, donde el } \\
\text { estudiante es protagonista del proceso de aprendizaje }\end{array}$ \\
\hline
\end{tabular}

Nota: Elaboración propia (2010). 
Esta especie de metamorfosis toma mayor relevancia al compararla con lo que la sociedad espera del educador en el proceso educativo con sus estudiantes. Casi en todos los sistemas educativos hay consenso en que la formación del ser humano, tarea a cargo, principalmente, de los profesionales en educación, debe propiciar personas íntegras, con visión holística del mundo, con altos principios éticos, críticos, autorrealizadas, capaces de aprender a vivir en sana convivencia, con una rica vida espiritual.

En Costa Rica, por ejemplo, esta aspiración se encuentra plasmada en la Ley Fundamental de Educación y en La Política Educativa hacia el siglo XXI; instrumentos con los cuales se guía el Ministerio de Educación Pública. Esta Ley fue aprobada desde 1994, específicamente, el 08 de noviembre, sesión No. 82-94, del Consejo Superior de Educación y dispone al respecto:

(...) La Política Educativa, amparada al Marco Jurídico vigente, propicia la búsqueda y concreción de un costarricense del siglo XXI que, consciente de las implicaciones éticas del desarrollo, sea PERSONA con rica vida espiritual, digna, libre y justa; CIUDADANO formado para el ejercicio participativo de la democracia, con identidad nacional, integrado al mundo, capaz de discernir y competir, autorrealizado y capaz de buscar su felicidad; PRODUCTOR para sí mismo y para el país desde el punto de vista de su condición de trabajador, lo que comporta el incremento de sus habilidades, el aprendizaje de destrezas y la búsqueda del conocimiento; SOLIDARIO por experimentar como propias las necesidades de los demás y, en consecuencia, con aptitud para buscar formas de cooperación y concertación entre sectores, velar por la calidad de vida de todos los ciudadanos y de las futuras generaciones, a partir de un desarrollo sustentable, ecológico y socialmente y CAPAZ DE COMUNICARSE CON EL MUNDO DE MANERA INTELIGENTE de tal manera que, a partir de la valoración de lo que lo identifica como costarricense, tome las decisiones que lo relacionen con otras culturas desde un punto de vista de pensador independiente, flexible y critico, teniendo por guía los derechos y los deberes humanos. (MEP, 1994, Declaraciones, párr. 1)

De manera que para favorecer la formación de la persona, según lo enunciado, son dignas de considerar las características mínimas que el perfil del docente requiere para llevar a cabo tan inconmensurable misión; dentro de las cuales sobresalen:

Visión flexible, holística de los desafíos emergentes socialmente.

$\checkmark$ Competencias profesionales que le capaciten para trabajar con tolerancia a la diversidad, en una sociedad multicultural.

$\checkmark$ Búsqueda permanente para la actualización de los saberes.

$\checkmark$ Habilidad para gestionar y aplicar el conocimiento en su práctica pedagógica.

$\checkmark$ Visión crítica transdisciplinaria.

$\checkmark$ Comprometido con el cambio y con la innovación

$\checkmark$ Habilidad para operar interconectado en redes de comunidades prácticas.

$\checkmark$ Consciente de la necesidad de conocer el contexto sociocultural y natural del estudiantado en un mundo complejo. 
Todas las transformaciones educativas que la desafiante sociedad de la información ha traído consigo no se conciben en el plano operativo sin considerar que llevan implícito un eje trasversal referente a las condiciones laborales de quienes tienen a cargo la misión más noble de una sociedad: la educación; son estos los profesionales en educación, con quienes los sistemas educativos, principalmente en América Latina, se encuentran en deuda respecto a la dignificación de su labor docente; masa profesional por sí misma y proporcionalmente estratégica para el desarrollo humano y social.

\section{Condiciones dignas de la labor docente, una revisión en distintos sistemas educativos}

Es casi una constante, especialmente en países de América Latina, como lo expone Parra (2005), que la labor docente se enfrenta a un exceso laboral que atenta contra la salud de esta población e incide en la calidad de los procesos de enseñanza que tiene a cargo. Costa Rica no es una excepción, no obstante los esfuerzos de parte de las autoridades educativas y, principalmente, por las asociaciones gremiales. El garantizar la dignidad profesional y humana de las profesionales y los profesionales de la educación es agenda prioritaria de las instancias gremiales, indistintamente de la región en que se encuentren.

Mediante un estudio realizado por la OREALC/UNESCO en Argentina, Chile, Ecuador, México, Perú y Uruguay (Parra, 2005) se encontró una serie de variables asociadas a las condiciones de los docentes, muy parecidas entre distintos países en las que se encuentran:

La mayoría de sus docentes destinan más de 30 horas semanales al trabajo, tomando en cuenta los tiempos dentro y fuera de su jornada debidamente remunerada.

En todos los países la carga de trabajo fuera del horario es alta, excepto en Perú. También en la mayoría de los países involucrados en el estudio sucede que actividades concernientes a preparar las clases, el material didáctico, actividades extraprogramáticas y asistencia a cursos de perfeccionamiento se realizan fuera del horario de trabajo.

En algunos países se reporta que la planificación en equipo, el trabajo administrativo y la atención a padres de familia se desarrollan fuera de la jornada laboral. Se evidencia cómo el trabajo invade los espacios extralaborales, dejando muy poco tiempo para descansar. A lo que se une, en gran dimensión, las horas dedicadas al trabajo doméstico, por ser la masa laboral, principalmente, femenina.

Un porcentaje elevado también reportó tener otro trabajo remunerado. El estudio evidenció que sumando el trabajo docente, el doméstico y el adicional, es evidente una sobrecarga laboral en la que se le cede poco tiempo al descanso, lo cual se constituye en un factor de riesgo de la salud del docente y de la calidad de la educación que ofrece.

Sobresale en el mismo estudio, como factor crítico y común en los distintos países participantes, la falta de espacio para que los profesores y profesoras se dediquen a la preparación de materiales y clases. También la falta de espacios para el descanso y servicios básicos. Por la escasez de espacios para actividades como planeamiento y elaboración de materiales, se asume como normal hacer este tipo de trabajo en el hogar. Los materiales de trabajo, igualmente, se consideran insuficientes y no pertinentes. Muchos docentes aportan ellos mismos los materiales con que trabajan.

También resultó en el estudio la variable ergonómica en forma significativa, así quedó evidenciado que los docentes esfuerzan, constantemente, la voz, y el trabajo requiere de la exigencia 
de permanecer de pie. Estas características son comunes en los países en estudio en un $42 \%$ y sobre el $90 \%$, respectivamente.

En todos los países se considera como el principal obstáculo para su desempeño, el excesivo número de estudiantes por salón de clase y la falta de especialistas para ofrecer apoyo en la escuela. En este rubro también sobresalió la falta de apoyo de padres y tutores, y las condiciones de infraestructura. En el perfil patológico es evidente que el docente se expone a enfermedades de carácter estacional como los resfríos, enfermedades respiratorias, al estar expuestos a un gran cantidad de estudiantes (Parra, 2005).

Según la Oficina Regional de Educación de la UNESCO para América Latina y el Caribe (OREALC), al exponer un estudio sobre las condiciones de trabajo y salud docente, con el afán de comprender su situación laboral y su incidencia en el desempeño, sobresale el hecho de que es una profesión donde se acepta, ampliamente, que parte del trabajo docente se realiza fuera de la jornada laboral; es decir, en horas que se suponen son para descanso o actividades personales del educador (VII Reunión del Comité Regional Intergubernamental del Proyecto Principal de Educación. Declaración de Cochabamba, 2001)

Hay un estudio interesante, específicamente el estudio PISA, en 44 países, cuatro de ellos latinoamericanos. Se evidenciaron algunas variables que están, claramente, relacionadas con el desempeño de los docentes y el rendimiento educativo. Estas son: clima en el aula, autoestima del docente y expectativas que tienen los docentes respecto de sus estudiantes (Robalino, 2005).

La realidad costarricense no es muy diferente; un estudio realizado por el Instituto de Investigación en Educación (INIE) (Cordero, Molina, Páez y Vargas, 2010), en escuelas públicas y subvencionadas, evidenció que los docentes de primaria enfrentan sobrecarga laboral, debido a que emplean su tiempo libre para llevar a cabo distintas tareas propias de su trabajo, sin ningún tipo de remuneración. Algunas de estas actividades responden a compromisos del Ministerio de Educación Pública mediante convenios y otras son meramente administrativas y relacionadas con el proceso de enseñanza-aprendizaje que dirigen. También se evidenció que las mujeres experimentan una triple jornada laboral de forma no remunerada, además de las funciones dentro de su horario y las que se llevan a cabo fuera de este, las tareas domésticas vienen a engrosar este rubro, pues la mayoría de profesionales en el sector educativo son mujeres. El estudio evidenció que el docente enfrenta niveles de cansancio emocional. Es importante tomar en cuenta que se sienten realizados, personalmente, en su labor, lo que les hace manifestar competencia y eficacia en su desempeño. Se evidenció que estos profesionales perciben una desvalorización social de su labor.

\section{Dignidad en las condiciones laborales del profesional en educación}

La palabra dignidad, etimológicamente, tiene su origen en el latín dignǐtas, -ātis, que se refiere a excelencia, realce, decoro de las personas en la manera de comportarse, cargo o empleo honorífico y de autoridad.

El concepto de dignidad se asocia con el derecho a ser tratado apropiadamente, donde prevalece el respeto. Se vincula también y de manera importante con la ética. Se dice que algo es digno cuando es valioso. Todas las personas, por el hecho o condición de ser seres humanos, tienen 
dignidad y valor inherentes. Se presenta como un llamado al respeto incondicionado y absoluto, y debe extenderse a todos (Vidal, s. f.).

Las condiciones de trabajo son conceptualmente definidas como “(...) el amplio escenario donde convergen un conjunto de dimensiones sociales, personales y físicas en las cuales laboran los docentes (...)" (Robalino, 2005, p.16). Esta autora llama la atención acerca de que, asociado a las condiciones de trabajo, también hay condiciones relacionadas con la salud del docente que dependen de un equilibrio social, biológico, psicológico y fisiológico, concernientes a la forma en que se desempeñan. Este es un tema digno de considerar, cuando se discute sobre el nivel de desarrollo humano que se aspira mediante una educación de calidad.

Al hablar de condiciones dignas de la labor docente, se debe partir de un principio inalienable de la profesión del educador. Este es la consideración de esta como una profesión de alto valor; quienes la ejercen merecen, socialmente e institucionalmente, el derecho a ser tratados como profesionales, al igual que aquellos dedicados a las otras disciplinas.

Siguen siendo insuficientes los esfuerzos por la dignificación del educador como profesional, para que opere en ambientes laborales adecuados, según las exigencias de su rol ante una sociedad desafiante en constante cambio. El tema de las condiciones dignas de la labor docente requiere de una constante reflexión, debido al carácter inestable de las transformaciones sociales hacia las que actúa.

Las condiciones laborales del educador deberían determinarse en busca de lograr una enseñanza eficaz, con el fin de permitir que estos profesionales se entreguen, plenamente, a sus tareas como profesionales de la educación. A su vez, debe ser una profesión reconocida en la sociedad, como una fuerza que contribuye, significativamente, al progreso de la educación, por lo debe contar con la posibilidad de participar, activamente, en las políticas de su profesión (UNESCO-OIT, 1996).

En la Conferencia Intergubernamental Especial sobre la Situación del Personal Docente, se hace referencia a la realidad de este personal y se destaca, entre muchas condiciones, la importancia de reconocer que el progreso de la educación depende, en gran medida, de la formación y la competencia del profesorado, incluyendo sus cualidades humanas, profesionales y pedagógicas. También se reconoce que la situación del profesional en educación debería ser consecuente con las exigencias de la educación, así como que la profesión docente tiene derecho a disfrutar del merecido respeto público (UNESCO-OIT, 1996).

Las condiciones dignas, según las exigencias del nuevo orden mundial, han traído a la discusión, el repensar el rol del docente en los aprendizajes de los nuevos tiempos. Es evidente el cambio conceptual que se está dando respecto del papel del educador (Robalino, 2005). También, las formas de aprendizaje han cambiado, las trasnformaciones en las que la sociedad del conocimiento ha traído consigo nuevas formas de aprendizaje y nuevas demandas al profesional en educación. En este marco, se ha abordado la identificación de una serie de indicadores que determinan el desempeño del docente, donde no solo se concibe importante los programas de capacitaciones y las políticas de salarios, para que los cambios propuestos en las reformas educativas sean posibles, sino también existe consenso sobre el hecho de que si no se cuenta con condiciones laborales dignas para la ejecución de las reformas educativas, no hay reforma educativa que alcance los niveles esperados, desde la perspectiva con la cual se planteó.

Diferentes estudios en distintas disciplinas coinciden en la influencia de las condiciones de trabajo y la salud en el rendimiento laboral. Sin embargo, en el campo educativo no son muchos los estudios. Una de las razones se debe a que, históricamente, a la docencia se le ha concebido como un apostolado que 
implica sacrificio, más que un trabajo para el cual se requieren estándares de desempeño. El debate de la profesionalización docente es relativamente nuevo, el cual surge ante las discusiones sobre la calidad de la educación y esta como eje estratégico para el desarrollo (Robalino, 2005).

Históricamente, se consideró que para ejercer la docencia bastaba con un sentido de vocación, no eran necesario conocimientos complejos, ni formación profesional permanente, se pensaba como un trabajo de medio tiempo que, a criterio de Robalino (2005), podría relacionarse con la clara incursión de la mujer en relación con el hombre, en este campo. Hoy, en la postmodernidad, este paradigma ha sido superado, gracias a los esfuerzos de muchos educadores y asociaciones gremiales abocadas a dignificar la labor docente como aquella profesión de formación continua, de habilidades para desempeñarse en ambientes complejos e inciertos, y con alta sensibilidad humana.

El sistema educativo costarricense ha llevado a cabo importantes esfuerzos en el mejoramiento de la condición de los docentes. El Ministerio de Educación Pública considera, como acciones relevantes, la capacitación y la actualización docente, al igual que el apoyo de la labor de estos profesionales en campos estratégicos de renovación curricular, para lo cual se hace uso de convenios con universidades (MEP, 2006).

En Costa Rica, el Consejo Nacional de Rectores [CONARE] (2006) reconoció como tarea fundamental atender a los educadores en su formación, selección y constante actualización, de manera que su trabajo les permita la realización como personas que son, que se encuentren en forma permanente motivados, eso sí, sin descuidar el proveer a estos profesionales de condiciones de trabajo aceptables.

El trabajo de los profesionales de la educación de este país ${ }^{2}$ representa, para Costa Rica, una importante y estratégica fuerza laboral, proporcionalmente significativa, como medio para lograr los avances del desarrollo humano y social a que aspira esta nación. Son muchas las razones obvias para considerar, reflexionar y definir condiciones dignas de la labor docente Estos deberían ser los profesionales mejor atendidos socialmente, lo cual no significa menospreciar otras disciplinas, sino un reconocimiento al valor incalculable que representan en el desarrollo tecnológico, económico, cultural y social.

Es importante hacer mención de una serie de indicadores de los que hay consenso en distintos sistemas educativos y que se consideran condiciones dignas para el desempeño del profesional en educación.

- Horarios: resulta indispensable contar con horarios de trabajo aptos para el proceso de enseñanza y aprendizaje. Los sistemas educativos deben considerar invertir en infraestructura y recurso humano para contar con horarios organizados en las primeras horas del día. No obstante, hay que reconocer que, en el sistema educativo costarricense y otros, se han hecho importantes esfuerzos al respecto, a pesar de la complejidad de este rubro. La jornada ideal es una sola, inicia aproximadamente a las siete de la mañana. Los horarios de doble jornada traen consigo agotamiento tanto del estudiantado como del profesorado. Diversas son las razones de este hecho, por ejemplo, el consumo de alimentos que hace que el metabolismo no se encuentre en mejores condiciones. En el sistema educativo costarricense ${ }^{3}$ y algo similar sucede en otros, los horarios se establecen en tres modalidades a saber: horario alterno, horario de dos jornadas y triple jornada:

\footnotetext{
En el sistema educativo costarricense los educadores al 2010 superan los 65.000 .

En el sistema educativo costarricense cada educador debe trabajar 29 horas y 20 minutos por semana, en dos jornadas de 14 horas y 40 minutos, o 22 lecciones de cuarenta minutos a la semana.
} 


\section{URL: http://www.una.ac.cr/educare}

Tabla 2

Modalidad de horario de la educación primaria

(I y II ciclos de la enseñanza general básica)

Sistema Educativo Costarricense

\begin{tabular}{|c|c|c|}
\hline Horario Alterno ${ }^{4}$ & Horario de dos jornadas por día ${ }^{5}$ & Horario de triple jornada ${ }^{6}$ \\
\hline $\begin{array}{l}\text { Centros educativos donde el } \\
\text { educador elabora con su grupo } 3 \text { días } \\
\text { con } 4 \text { lecciones (mañana: 7:00 a.m. a } \\
\text { 10:00 a.m./por la tarde 10: } 40 \text { a.m. a } \\
\text { 13:40 pm) y dos días con } 5 \text { lecciones } \\
\text { (horario de 7:00 am a 10:50 am/ por } \\
\text { la tarde 11:30 am. a 15:20 p.m.). Con } \\
\text { un total de } 22 \text { lecciones de cuarenta } \\
\text { minutos a la semana. }\end{array}$ & $\begin{array}{l}\text { Centros educativos donde se labora } \\
\text { con dos jornadas por día, con un } \\
\text { total de } 18 \text { horas laboradas y } 40 \\
\text { minutos. }\end{array}$ & $\begin{array}{l}\text { Centros educativos con triple } \\
\text { jornada y con jornada semanal } \\
\text { de seis días: se trabaja seis } \\
\text { días a la semana, el número } \\
\text { de lecciones oscila entre } \\
\text { veinticuatro y veintinueve } \\
\text { lecciones. }\end{array}$ \\
\hline
\end{tabular}

En el III ciclo de la Educación General Básica y en la Educación Diversificada, el profesional en educación puede optar por un máximo de 48 lecciones semanales, de estas 40 podrán ser en propiedad. Podrán ser distribuidas de acuerdo con el plan de estudios, con lecciones propias de la especialidad y con las lecciones "optativas", sin que ello signifique disminución de las lecciones en propiedad.

- Ejercicio profesional digno en zonas rurales: garantizar el ejercicio profesional digno en zonas rurales implica considerar fundamental que los docentes asignados a estas zonas cuenten con condiciones dignas mínimas de vivienda y opciones de capacitación.

- Ejercicio profesional en zonas de alta vulnerabilidad social o zonas de menor desarrollo: gracias a los esfuerzos gubernamentales y de asociaciones gremiales, hoy en Costa Rica, los educadores que trabajan en estas zonas cuentan con un incentivo económico adicional, el cual es digno de conservar.

- Participación activa en la definición de políticas educativas: en la medida en que a los educadores se les permita la participación activa en las políticas educativas, se puede asegurar que los intereses y demandas de este grupo profesional serán incorporadas. Al respecto, es importante que se sigan manteniendo los foros educativos y de participación comunal como espacios para la concertación comunal y la toma de decisiones correspondientes.

Escuelas con horario alterno: los maestros tienen a su cargo dos grupos y trabajan dos jornadas diarias. Cada educador debe laborar 29 horas y 20 minutos por semana, atendiendo a sus grupos en dos jornadas de 14 horas y 40 minutos cada una, lo que significa un total semanal de 22 lecciones de 40 minutos para cada grupo.

5 Escuelas con una o dos jornadas diarias: el maestro labora 18 horas y 40 minutos, es decir, 28 lecciones de 40 minutos, atendiendo en una jornada a un grupo de alumnos durante cinco días a la semana.

6 Escuelas con triple jornada y con jornada semanal de seis días: el módulo horario está conformado por una jornada laboral de seis días, en las que se imparte un número de lecciones académicas que puede oscilar entre 24 y 29; ese margen está condicionado por el número de lecciones complementarias que se imparten en cada centro educativo. La duración de cada lección es de 60 minutos. 
- Carga laboral justa: donde se libere al docente del exceso de trabajo administrativo para que pueda ofrecer una mejor calidad en su desempeño pedagógico, sin menoscabo de salud. Es importante que la carga laboral sea acorde con su jornada remunerada.

- Reconocimiento social: la labor docente debe ser reconocida por la sociedad como una carrera profesional.

- Remuneración justa: la profesión docente merece una remuneración adecuada, como las otras disciplinas. En este rubro se han hecho importantes esfuerzos de parte de las autoridades educativas y asociaciones gremiales; condiciones que requieren de un monitoreo constante.

- Reconocimiento del profesional en educación protagonista en el proceso de enseñanza: debe reconocerse como actor fundamental en el proceso de la enseñanza, no solo como ejecutor de los lineamientos políticos, sino también como persona con derecho a elaborar, adaptar los programas y los métodos de enseñanza adecuándolos a la realidad de su contexto, igualmente que participe en la elaboración de políticas de reforma por realizar.

- Capacitación permanente y pertinente: la capacitación debe ser más permanente, ajustada a las nuevas metodologías y técnicas modernas al lado del avance tecnológico. Los procesos de capacitación no deberían darse en tiempo libre del educador, principalmente. Estos programas de capacitación deben continuar acompañándose de incentivos profesionales en la carrera docente. Es importante valorar la inversión de contar con personal especializado de apoyo, para que atienda a los estudiantes mientras los docentes se capacitan dentro de su jornada laboral, de esta forma no se abandona el programa curricular estudiantil. Disponer de tiempo suficiente para participar de actividades de crecimiento profesional durante el ejercicio de su cargo debe ser, independientemente de si se desempeña en una zona rural o marginada, un incentivo. Las condiciones dignas empiezan con una competitiva formación profesional, un docente con una formación académica de calidad sabrá determinar las condiciones óptimas para su eficaz desempeño.

- Sistema de incentivos: se debe ofrecer al docente mejores incentivos que lo motiven a trabajar con más dedicación, ya que si las escuelas y colegios están en mal estado, sin mobiliario adecuado, les falta bibliotecas y otros recursos, se sentirá desmotivado. Entonces, debe haber, por parte de la administración gubernamental, estímulos como: una dinámica capacitación, cursos especiales, becas, programas de apoyo o cooperación técnica, seminarios, ofrecer el año sabático a los docentes, eliminar cargos administrativos que no les corresponden, disposición de licencias de estudio tanto en el ámbito nacional como en el internacional. Se deben considerar condiciones para participar de intercambios, pasantías en otros sistemas educativos. Estas posibilidades traen implícito un valor agregado que representa un crecimiento de esta modalidad. Dentro de los incentivos se encuentra la posibilidad de contar con un sistema de ascensos sobre una evaluación objetiva del desempeño, según criterios profesionales estrictamente determinados por las autoridades correspondientes, en función de sus méritos académicos. Contar con un atractivo y justo sistema de jubilación, también entra en el rubro de incentivos.

- Apoyo ministerial a la imagen del profesional en educación: el Ministerio de Educación Pública debe aunar mayores esfuerzos por realzar el liderazgo y la imagen positiva del docente. Promover una campaña por la dignificación del docente, no solo mejorando salarios, sino 
favoreciendo un mejor clima laboral en las instituciones, iniciando desde las oficinas centrales. Hay que luchar por mejorar el prestigio y autoestima de los profesionales de la educación, condiciones que hace mucho se han socavado por las mismas autoridades de gobierno.

- Ley de Carrera Docente: es una importante ley que se requiere actualizar para poder ponderar otras especialidades de la Carrera Docente.

- El tema salarial: seguirá siendo en el siglo XXI de agenda en los gremios de la educación y en las políticas públicas en materia educativa. El profesional de la Educación deberá continuar trabajando para colocar su profesión sin distinción, salarialmente, de otras profesiones.

- Organización de los grupos de estudiantes en el salón de clases: hay consenso en que el tamaño ideal en los salones de clase es de grupos con un máximo de 25 alumnos, o bien inferior a este número. Sin embargo, dadas las condiciones con que cuentan la mayoría de administradores de la educación, este rango no ha sido posible en la práctica, por la exagerada demanda del servicio educativo, aunada a las condiciones de infraestructura, que obligan a la administración a conformar grupos de más de 25 alumnosn con los agravantes que esta situación implica.

- Adecuaciones curriculares: si bien es cierto, en el sistema educativo costarricense existen leyes como la 7600, que hace referencia a las inclusiones de los estudiantes con necesidades educativas especiales al sistema formal, en las organizaciones educativas se opera en tensos ambientes relacionados con las adecuaciones curriculares significativas, adecuaciones curriculares no significativas y las adecuaciones curriculares de acceso. Desde la administración de la educación, este tema no es fácil en su ejecución, al menos se debería considerar que cuando en un salón de clase exista un cantidad determinada de estos casos, lo ideal es que se baje el rango de matrícula por grupo, o bien, se cuente con personal de apoyo especializado para actividades relacionadas con la integración de alumnos con necesidades educativas especiales. Es un apoyo interdisciplinario efectivo. Para efectos de planificación presupuestaria, esta realidad no es una norma. Es importante, al respecto, considerar el tema de personal especializado de apoyo.

- Estabilidad laboral: para todo trabajador es una aspiración gozar de estabilidad laboral; sin embargo, esta es una realidad que aflige a distintos profesionales en educación en diferentes sistemas educativos. En Costa Rica existe un fallo judicial en la Sala Constitucional, el cual ordena la estabilidad laboral a todos aquellos funcionarios que ocupen cargos en forma interina, no obstante, por razones particulares, esta obligatoriedad no es una realidad para todos. Es importante considerar, en este rubro, el tema de la necesidad de que los profesionales de la educación no pertenezcan a distintos centros educativos para completar sus horas, debido al desgaste que implica esta condición, tanto para él como para la calidad de la educación que ofrece.

- Espacios físicos aptos para el desempeño profesional: es una necesidad que los educadores dispongan de espacios aptos para preparar clases y materiales didácticos. Para el uso de estos, lo ideal es que el docente cuente con jornada remunerada para dedicarlos a este tipo de actividad académica como parte de su desempeño; ya que, en algunos casos, existen centros educativos que cuentan con estas áreas, sin embargo, los educadores alegan no tener tiempo para ello. A su vez, se deben considerar espacios físicos que permitan la convivencia del personal docente. Dentro 
de este rubro, son considerados ambientes escolares de aprendizaje dotados de formas físicas y recursos tecnológicos pertinentes.

- Infraestructura adecuada: debe ser apta, según las demandas de los tiempos, salones de clases y centros de información acordes con las nuevas formas de aprendizaje de sus alumnos y las nuevas formas de enseñar de los docentes. Esta condición requiere dotar los centros educativos de recursos pedagógicos de punta, según las exigencias de la potmodernidad.

- Recursos pedagógicos y capacidad tecnológica para acceder a las tecnologías de la información: lo ideal es que todos los centros educativos cuenten con acceso a las tecnologías de la información y la comunicación, para el servicio del proceso de enseñanza a su cargo. Para el uso eficiente de estos recursos, se requiere el entrenamiento correspondiente. En el siglo XXI, no debe ser un problema del educador el disponer de los recursos pedagógicos mínimos y actualizados para llevar a cabo su labor.

\section{Dimensiones de las condiciones dignas de la labor docente}

La labor docente por sí misma es ambivalente, tiene una dimensión profesional apasionante y tiene una dimensión referente a las condiciones laborales con las que se lleva a la práctica, la cual, en muchos casos, no es la óptima y atenta con los principios nobles a que aspira la educación. Esta es una labor profesional que para su éxito y ejecución no depende, únicamente, de los conocimientos adquiridos profesionalmente, tiene un fuerte componente determinado por las condiciones laborales en las que se debate el docente diariamente. Estas se pueden dividir en tres grandes grupos, a saber: cognitiva, operacional e institucional.

Dimensión cognitiva: esta área se refiere a las competencias profesionales que capacitan al profesional en educación para poder desempeñarse con amplio dominio teórico y práctico en su profesión. La calidad de la formación profesional que posee es fundamental, así como los procesos de educación permanente.

Dimensión operativa: se refiere a todas las condiciones institucionales relacionadas con infraestructura, recursos pedagógicos, número de estudiantes por aula y el acceso a las tecnologías de la información y la comunicación al servicio del proceso de la enseñanza y el aprendizaje. También incluye elementos asociados a las zonas geográficas y condiciones sociales en las que opera la institución, las cuales limitan la labor docente en gran medida.

Dimensión institucional: se refiere a los ambientes laborales generados en las instituciones, tales como el clima organizacional, cultura organizacional, relaciones humanas, sistemas de comunicación y tipo de liderazgo. Todos estos elementos limitan o favorecen las condiciones dignas del desempeño del profesional en educación. 

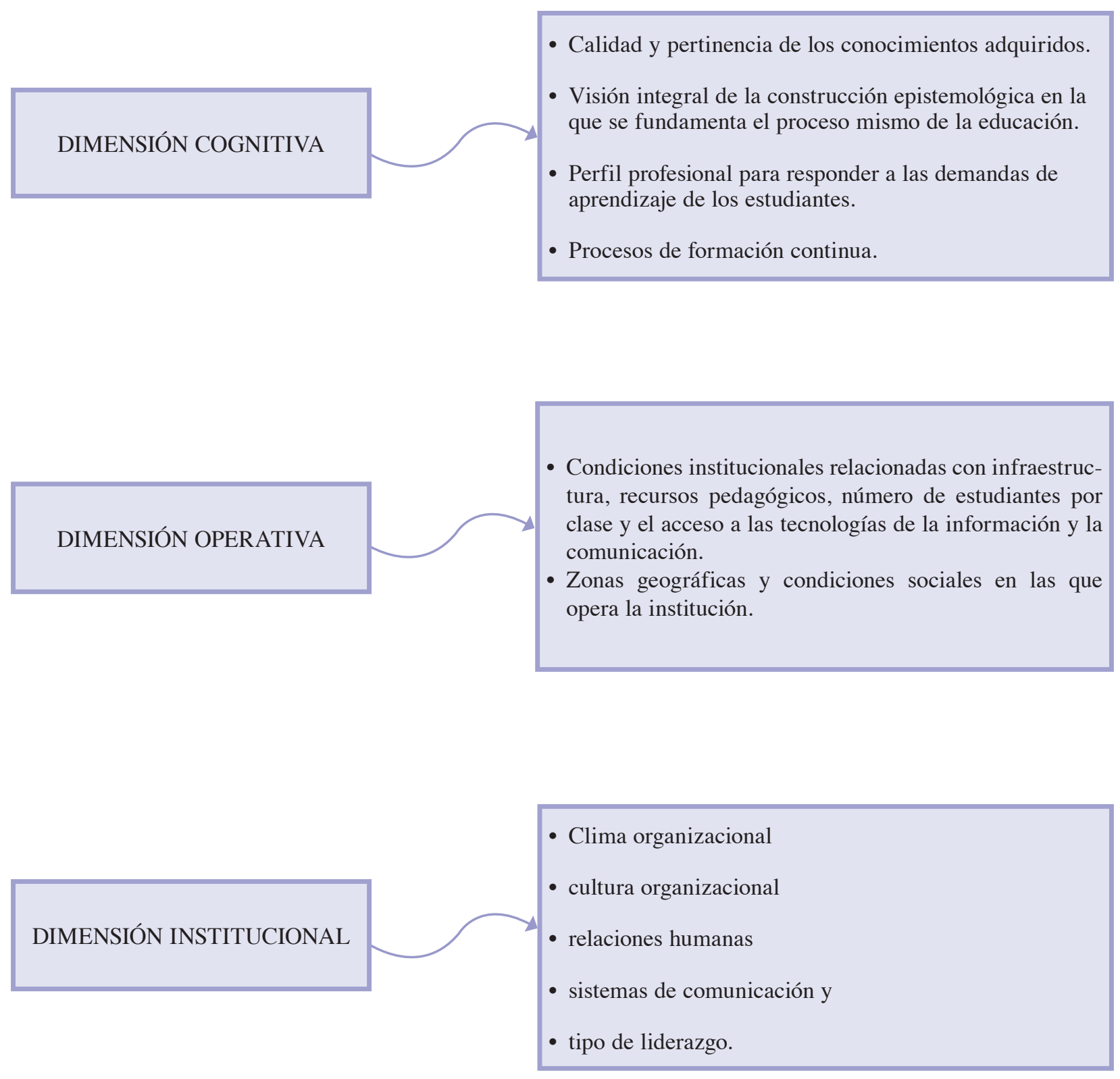

Figura 1. Dimensiones del desempeño laboral digno del profesional en el campo de la educación.

Comprender la complejidad en la que actúa el profesional en educación en la postmodernidad, implica, a su vez, reconocer la responsabilidad que tiene la sociedad con las condiciones laborales en que este se desempeña, de manera que la enseñanza a su cargo sea ejecutada según la calidad de las exigencias de la nueva sociedad emergente. No se puede hablar de profesionales en educación competitivos, según las exigencias de las transformaciones sociales, sin reconocer que su calidad en el desempeño tiene que ir de la mano con escenarios laborales dignos. 


\section{Consideraciones finales}

A manera de síntesis, la dignidad del profesional en educación, sus condiciones laborales y necesidades han sido temas de especial preocupación de muchas organizaciones. El sistema educativo costarricense cuenta con diferentes asociaciones gremiales que han hecho importantes esfuerzos en esta materia ${ }^{7}$. Todas estas agrupaciones gremiales buscan mejorar las condiciones académicas y económicas de sus miembros y colaboran, ampliamente, con los cursos de capacitación y formación para los profesores en servicio.

La calidad de la educación y la calidad y dignidad del personal docente son factores que están correlacionados y son importantes para un adecuado desempeño del sistema educativo. En esta línea se reafirma que es importante dejar claro que las condiciones labores dignas de la labor docente no pueden concebirse exclusivamente como políticas salariales, excluyendo importantes temas que tienen una relación directa con la calidad de vida del profesional en educación, como lo son los componentes afectivo, humano, social, y espiritual que inciden en su desempeño como educador. También hay una dimensión cognitiva, que tiene que ver con la formación profesional y programas de formación continua. Durante mucho tiempo, se consideró la dimensión cognitiva como la única, la cual tiene que ver con el perfil profesional en educación, que lo habilita en forma eficiente en su desempeño.

La otra dimensión es la operativa, asociada con la infraestructura, la distribución de los estudiantes en los salones de clase, el acceso a recursos tecnológicos. También incluye elementos relacionados con las zonas geográficas y las condiciones sociales en las que opera la institución.

La última dimensión es institucional, se refiere a los ambientes laborales generados en las instituciones, tales como aspectos relativos al clima organizacional, la cultura organizacional, las relaciones humanas, sistemas de comunicación y tipo de liderazgo.

Las condiciones dignas de la labor docente han sido discutidas en diversos foros a escala mundial en distintos momentos y contextos, y persiste como un tema no agotado en el ámbito global. Es un contenido que obliga a pensar en nuevas estrategias y en la búsqueda de consenso de políticas integrales que favorezcan las condiciones laborales de la labor docente; condiciones que están articuladas, en gran medida, con la calidad de aprendizaje que se ofrezca. Las características en las que se desempeña el docente son fundamentales para una educación inclusiva de calidad. Al respecto, el Estado tiene el deber de proporcionar ambientes laborales que faciliten el proceso de la enseñanza y el aprendizaje.

El tema de las condiciones dignas de la labor docente debe ocupar un lugar prioritario en las agendas de política educativa, ellas son fundamentales en el desarrollo humano que se desea lograr en la exigente sociedad del siglo XXI. Esto implica desarrollar estrategias que reivindiquen la labor docente en forma holística. Para ello es fundamental el aporte de gremios de la educación que han liderado importantes luchas en la dignificación del desempeño docente; sin embargo, el siglo XXI promete un escenario complejo, donde emergerán nuevos desafíos, no menos exigentes que los actuales. Este tema seguirá siendo agenda en las negociaciones con los profesionales de la educación y los gobiernos, no es un tema acabado.

ANDE: Asociación Nacional de Educadores Costarricenses.

SEC: Sindicato de Educadores Costarricenses, fundada en 1969.

UCEP: Unión de Conserjes de la Educación Pública.

SINAPRO: Sindicato Nacional de Profesores de Orientación.

ADEP: Asociación de Educadores Pensionados.

ADEM: Asociación de Directores de Enseñanza Media.

SADEM: Sindicato de Asistentes de Dirección de Enseñanza Media. 
Es importante cuidar la labor docente, no solo se puede hablar de estudiantes marginados, también grupos de docentes que trabajan en condiciones marginales, abandonados de su propio ente, lo que hace que se tengan diferenciaciones entre docentes, según sus condiciones. El nuevo siglo no puede darse el lujo de desperdiciar el recurso docente en ningún tipo condición.

La discusión está clara, parece no haber duda de la dignificación laboral de los profesionales en educación; no obstante, sí hay fisuras acerca de la forma de lograrlo, razón por la cual las asociaciones gremiales y autoridades educativas requieren buscar espacios de diálogo en forma constante, en aras de una mejor educación cada día.

\section{Referencias bibliográficas}

Aguerrondo, I. (2007). Racionalidades subyacentes en los modelos de planificación (educativa). Buenos Aires, Argentina. UNESCO/IIPE. Recuperado de http://www.fhuce.edu.uy/academica/ cienciasEducacion/soc-econEducacion/planificacioneducativa/materiales/Materiales\%20 Planificacion/Ines\%20Aguerrondo\%20Racionalidades\%20Subyacentes.pdf

Bazarra, L., Casanova, O. y García, J. (2005). Ser profesor y dirigir profesores en tiempos de cambio ( $2^{\circ}$ ed.). Madrid, España: Narcea.

Bruner, J. S. (1988). Desarrollo cognitivo y educación. Madrid: Morata.

Castells, M. (noviembre, 2007). El papel de la creatividad y la innovación en la era del conocimiento. En Fundación Omar Dengo. Conferencia llevada a cabo en la Celebración del 20 aniversario de la Fundación Omar Dengo. San José, Costa Rica.

Chiavenato, I. (2002). Administración en los nuevos tiempos. Bogotá, Colombia. McGraw-Hill.

Consejo Nacional de Rectores [CONARE]. (2006). Hacia un modelo educativo para elevar localidad de la educación costarricense. Una propuesta de políticas, estrategias y acciones. San José, Costa Rica: UNED. Recuperado de http://www.conare.ac.cr/docs/mod_educ 2006/ CONARE 00.pdf

Cordero, R., Molina, N., Páez, W. y Vargas, Y. (2010). Análisis de las funciones y valoración de la carga laboral del puesto docente a nivel de primaria, de las agremidas y los agremiados de la Asociación Nacional de Educadores (ANDE) (Informe final). San José, Costa Rica: INIE, Universidad de Costa Rica.

Darling-Hammond, L. McLaughliln, M. (2003) El desarrollo profesional de los maestros. Nuevas estrategias y políticas de apoyo. Cuadernos de discusión, 9, 7-31. Recuperado de http://ses2. sep.gob.mx/dg/dgespe/cuader/cuad9/cuad9.pdf

Delors, J., Mufti, I., Amagi, I., Carneiro, R., Chung, F., Geremek, B., ... y Nanzhao, Z. (1996). La educación encierra un tesoro. Informe a la UNESCO de la Comisión Internacional sobre la Educación para el siglo XXI (Compendio). Francia: Santillana Ediciones UNESCO. 
Flores, R. (2000). Evaluación pedagógica y cognición. Colombia: McGraw-Hill.

Ministerio de Educación Pública [MEP]. (1994). Política Educativa hacia Siglo XXI. San José, Costa Rica: Autor.

Ministerio de Educación Pública [MEP]. (2006). Programa regional de mejora de la calidad educativa. Plan de acción de la educación. Programa de mejora de la calida educativa, Costa Rica. Proyecto para la mejora de la oferta, cobertura y alcance de la formación permanente de docentes y administradores de la educación. San José, Costa Rica: Autor. Recuperado de http://ceccsica.org/programas-accion/educa/paises/costarica.html

Ministerio de Educación Pública [MEP]. (2010). Nuestras diez líneas estratégicas: 2010-2014. Al desarrollo por la educación. Documento, Ministerio de Educación Pública, Administración Chinchilla: 2010-2014. San José, Costa Rica.

Parra, M. (2005). Estudio de caso en Chile. En OREAL/UNESCO (Aut.), Condiciones de trabajo y salud docente. Estudios de casos en Argentina, Chile, Ecuador, México, Perú y Urugay (pp. 75-102). Santiago, Chile: OREALC/UNESCO. Recuperado de http://www.oei.es/docentes/ publicaciones/condiciones trabajo_salud_docente.pdf

Programa Regional de Mejora de la Calidad Educativa. Plan de Acción de la Educación. Programa de Mejora de la Calidad Educativa 2000-2006

Robalino, M. (2005). Las condiciones de trabajo y salud docente: aportes, alcances y límites del estudio. En OREAL/UNESCO (Aut.), Condiciones de trabajo y salud docente. Estudios de casos en Argentina, Chile, Ecuador, México, Perú y Urugay (pp. 13-20). Santiago, Chile: OREALC/UNESCO. Recuperado de http://www.oei.es/docentes/publicaciones/condiciones trabajo salud docente.pdf

VII reunión del Comité Regional Intergubernamental del Proyecto Principal de Educación. Declaración de Cochabamba (2001). Revista Latinoamericana de Estudios Educativos, 31(2), 113-118. Recuperado de redalyc.uaemex.mx/pdf/270/27031206.pdf

UNESCO-OIT. (1996). Recomendación conjunta de la OIT y la UNESCO relativa a la situación del personal docente (1966). En UNESCO-OIT (Aut.), Recomendación de la OIT y la UNESCO relativa a la situación del personal docente (1966) y Recomendación de la UNESCO relativa a la condición del personal docente de enseñanza superior (1977) (pp. 21-50). París, Francia: Autor. Recuperado de unesdoc.unesco.org/images/0016/001604/160495s.pdf

Vidal, J. (s. f.). Valores y principios. La dignidad humana y sus implicaciones éticas. Recuperado de http://www.aceb.org/v_pp.htm 